\title{
Aconselhamento pré-viagem aos voluntários com destino ao Haiti. Relato da experiência do Ambulatório dos Viajantes do Hospital das Clínicas da FMUSP
}

\author{
Pre travel advice among volunteers to Haiti. Experience at \\ Travellers' Clinic of HCFMUSP
}

\begin{abstract}
Karina Takesaki Miyajii', Tânia do Socorro Souza Chaves², Amanda Nazareth Lara ${ }^{1}$, André Machado Luiz ${ }^{1}$, Ana Marli Christovan Sartori' ${ }^{1}$, Marta Heloisa Lopes ${ }^{3}$
\end{abstract}

Miyaji KT, Chaves TSS, Lara NA, Luiz AM, Sartori AMC, Lopes MH. Aconselhamento pré-viagem aos voluntários com destino ao Haiti. Relato da experiência do Ambulatório dos Viajantes do Hospital das Clínicas da FMUSP / Pre travel advice among volunteers to Haiti. Experience at travellers' clinic of HCFMUSP. Rev Med (São Paulo). 2014 abr.-jun.;93(2):90-3.

RESUMO: INTRODUÇÃO: Desastres naturais representam ameaça à vida e às condições mínimas de sobrevivência de milhares de pessoas. Em 12 de janeiro de 2010 o Haiti foi assolado por um dos mais devastadores terremotos da história do país. Equipes de resgate do mundo inteiro chegaram ao país para ajuda humanitária. O Ambulatório dos Viajantes, da Divisão de Clínica de Moléstias Infecciosas e Parasitárias da Faculdade de Medicina da Universidade de São Paulo (HC-FMUSP) fez a orientação pré-viagem dos voluntários que foram encaminhados ao serviço. OBJETIVO: Enfatizar a importância da medicina de viagem em emergência de saúde pública e descrever a orientação pré-viagem fornecida aos viajantes. MATERIAL E MÉTODOS: Estudo descritivo de relato da experiência do Ambulatório dos Viajantes da Divisão de Clínica de Moléstias Infecciosas e Parasitárias da FMUSP, na orientação pré-viagem de viajantes com destino ao Haiti. Toda a equipe profissional do ambulatório foi informada da situação e uma estratégia foi criada para atender a demanda sem comprometer a rotina. Orientação sobre os riscos de adoecimento através de água e alimentos, vetores, poluição do ar, animais peçonhentos, choque elétricos foram discutidos além da informação sobre os aspectos geográficos, históricos, políticos e econômicos. RESULTADOS: Foram atendidos 98 viajantes. A média da idade foi 38,9 (23 a 66) anos e 79,6\% eram do sexo masculino. Entre as ocupações referidas pelos viajantes atendidos foi possível observar: profissionais de saúde e bombeiros (52\%), técnicos de telefonia $(17,3 \%)$, policiais e militares $(16,3 \%)$ e outros profissionais $(14,2 \%)$. Vacinas indicadas e realizadas no serviço: febre tifóide $(92 \%)$, hepatite A $(87 \%)$, febre amarela (46\%), tétano e difteria (dT) (36\%), sarampo, caxumba e rubéola $(28 \%)$, poliomielite $(19 \%)$, vacina antimeningocócica conjugada C (6\%), influenza e raiva. Todas as medidas de prevenção para a malária foram recomendadas. Cloroquina foi a droga de escolha para a quimioprofilaxia da malária, e a ciprofloxacina para diarreia do viajante. Conclusão: A MV se faz presente a cada dia entre nós e ultrapassa barreiras em situações de emergências em saúde pública, como no caso de desastres naturais.

DESCRITORES: Ajuda humanitária; Haiti; Viajantes; Vacina.

1. Médica(o) Assistente do Centro de Referência para Imunobiológicos Especiais do Hospital das Clínicas da Faculdade de Medicina da Universidade de São Paulo (CRIE - HC FMUSP) e Ambulatório dos Viajantes da Divisão de Moléstias Infecciosas e Parasitárias, do Hospital das Clínicas da FMUSP. E-mail: karinamiyaji@gmail.com.

2. Ex-Médica Assistente do Centro de Referência para Imunobiológicos Especiais do Hospital das Clínicas da Faculdade de Medicina da Universidade de São Paulo (CRIE - HC FMUSP) e Ambulatório dos Viajantes da Divisão de Moléstias Infecciosas e Parasitárias, do Hospital das Clínicas da FMUSP. Pesquisadora em Saúde Pública. Seção de Parasitologia. Instituto Evandro Chagas, Secretaria de Vigilância em Saúde/Ministério da Saúde. E-mail: tania.chaves@uol.com.br.

3. Docente do Departamento de Moléstias Infecciosas e Parasitárias da Faculdade de Medicina da Universidade de São Paulo - FMUSP. E-mail: mahlopes@usp.br

Endereço para correspondência: Tânia do Socorro Souza Chaves. E-mail: tania.chaves@uol.com.br 
ABSTRACT: Natural disasters represent a threat to life and the minimum conditions of survival for millions of people can be compromised. On January 12, 2010 Haiti was ravaged by one of the most devastating earthquakes throughout its history. Rescue teams from around the world arrived in the country for humanitarian aid. OBJECTIVE: To emphasize the importance of travel medicine in emergency situations in international public health care and to describe the pre-travel medical advice provided to the travelers. MATERIAL AND METHODS: Retrospective analysis of records of travellers who sought pretravel medical advice at Travellers' Clinic of HCFMUSP in January, 2010 with travel to Haiti for Humanitarian Aid. All the professional staff of the clinic was informed about the action and in 24 hours the group created a strategy to address the cluster of fast and efficient way without compromising the routine of the clinic. General information about the aspects: cultural, historical, political and economic on Haiti were discussed with all volunteers. In the guidance on the

\section{INTRODUÇÃO}

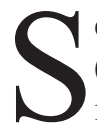
egundo a Organização Mundial de Turismo (UNWTO), pela primeira vez as viagens internacionais excederam a marca de um bilhão em 2012, com aumento de 995 milhões de viagens internacionais só em 2011 . A Medicina de Viagem (MV) surgiu nos países europeus e na América do Norte em resposta ao crescente deslocamento dos indivíduos para as mais diversas regiões do mundo com finalidades distintas, como o turismo, trabalho, estudo, missões militares e ajuda humanitária. Sua prática fundamenta-se na redução de riscos de adoecimento individual e coletivo, uma vez que a MV tem importante papel nas ações de controle da importação e exportação de doenças ${ }^{2,3,4,5,6,7}$.

A MV surgiu no Brasil no final da década de 90 coincidentemente com as reformas socioeconômicas e com as melhorias das condições de vida dos brasileiros. Em 1997, o primeiro serviço brasileiro especializado em medicina de viagem - o Centro de Informação em Saúde para Viajantes (CIVES) - foi criado pelo Departamento de Medicina Preventiva da Faculdade de Medicina da Universidade Federal do Rio de Janeiro. O Ambulatório dos Viajantes da Divisão de Clínica de Moléstias Infecciosas e Parasitárias do Hospital das Clínicas da Faculdade de Medicina da Universidade de São Paulo (HC-FMUSP) é um dos serviços pioneiros em nosso meio, dedicado ao ensino, à assistência, à pesquisa e constitui uma das referências em MV no Brasil ${ }^{3,8,9}$.

Nas últimas décadas o mundo tem acompanhado em tempo real desastres e catástrofes naturais, e como consequência tem aumentado o número de pessoas associadas ou não à organizações não governamentais interessadas em ajudar às vítimas. Ao mesmo tempo observa-se uma escassez de guias bem consolidados que orientam voluntários antes da viagem, ou estudos que analisam os voluntários que trabalharam voluntariamente em missões de ajuda humanitária ${ }^{10,11}$. prevention of infectious diseases, were emphasized: the risks of disease through food and water, vectors, animals and air pollution. RESULTS: 98 professionals were attended, 78 (79,6\%) male, the median age was 38,9 years old. Professionals categories: healthcare workers $(29,6 \%)$; military personal $(38,7 \%)$; phone technician $(17,3 \%)$ and others professionals $(14,2 \%)$. Recommended vaccines: typhoid fever (92\%), hepatitis A(87\%), yellow fever (46\%), dT (36\%), hepatitis B (36\%), SCR (28\%), polio (19\%), meningococcal vaccine, rabies and influenza. All preventive measures were recommended against malaria, chloroquine was the drug of choice for malaria chemoprophylaxis, and ciprofloxacin was the antibiotic of choice for travelers' diarrhea. CONCLUSION: The travel medicine is present every day between us and transcends barriers in unexpected situations such as emergency and rapid response in public health, as in the case of natural disasters.

KEYWORDS: Humanitarian aid; Haiti; Travelers; Vaccine.

No dia 10 de janeiro de 2010 o mundo acompanhou com perplexidade um dos maiores e devastadores terremotos que já assolou o Haiti, a cidade de Port-au-Prince, capital do país foi a cidade mais afetada. O governo do Haiti estimou cerca de 220.000 mil mortes e aproximadamente 300.000 feridos $^{12}$. A magnitude do desastre natural mobilizou voluntários de diversos países, inclusive o Brasil, e foram enviados grupos de profissionais para ajuda humanitária a fim de resgate, assistência às vítimas e para auxiliar na reconstrução do país. Alguns desses profissionais procuraram o Ambulatório dos Viajantes, da Divisão de Clínica de Moléstias Infecciosas e Parasitárias da Faculdade de Medicina da Universidade de São Paulo (HC-FMUSP) para orientação pré-viagem.

\section{OBJETIVO}

O objetivo do presente estudo é introduzir os princípios da MV e relatar uma das experiências do Ambulatório dos Viajantes, da Divisão de Clínica, de Moléstias Infecciosas e Parasitárias da Faculdade de Medicina da Universidade de São Paulo (HC-FMUSP), na atenção à saúde dos viajantes que se destinaram ao Haiti em ajuda humanitária por ocasião do terremoto que devastou o país em 2010.

\section{MATERIAL E MÉTODOS}

Trata-se de um estudo descritivo de relato da experiência do Ambulatório dos Viajantes da Divisão de Clínica de Moléstias Infecciosas e Parasitárias da FMUSP, na orientação pré-viagem de viajantes com destino ao Haiti em 2010. Após decisão das autoridades de governo para o envio de profissionais em missão de ajuda humanitária ao Haiti, o Ambulatório dos Viajantes do Hospital das Clínicas foi convocado para liderar o aconselhamento antes 
da viagem aos viajantes interessados em ajudar as vítimas no Haiti. Toda a equipe profissional do Ambulatório dos Viajantes foi informada sobre o fato, e uma estratégia para manter o atendimento de rotina e atender a demanda solicitada foi criada.

$\mathrm{O}$ atendimento de orientação pré-viagem consiste de consulta médica em que as medidas de prevenção gerais e de intervenção para reduzir os riscos de adoecimento são abordadas. Entre as medidas gerais de prevenção discutidas na consulta estão a forma de transmissão de agravos através da água e alimentos, vetores, poluição do ar, animais peçonhentos, choque elétricos; além de informações sobre os aspectos geográficos, históricos, político, econômicos e culturais relacionados ao destino. As medidas de intervenção são a vacinação e as profilaxias com fármacos que são indicadas de acordo com as características da viagem e os antecedentes do viajante. Todas estas informações constam da ficha de atendimento. A partir das informações contidas na ficha de atendimento, as variáveis foram indexadas em um banco de dados no programa Microsoft Excel e os dados foram analisados.

\section{RESULTADOS}

Foram atendidos 98 viajantes com destino para o Haiti no período de 15/01/2010 a 09/04/2010. A idade média foi de 38,9 (23 a 66) anos, 79,6\% dos viajantes eram do sexo masculino. Entre as ocupações referidas pelos viajantes atendidos foi possível observar: profissionais de saúde e bombeiros corresponderam $(52 \%)$, técnicos de telefonia $(17,3 \%)$, policiais e militares $(16,3 \%)$, outros profissionais $(12,2 \%)$ e $2 \%$ não referiram à profissão. Oitenta viajantes negaram ter doença de base, dez referiram doença cardiovascular, quatro relataram ter asma brônquica, três diabetes, três alterações de tireóide, dois relataram ansiedade/depressão e um referiu hepatite C crônica.

Vacinas indicadas e realizadas no serviço: febre tifóide (92\%), hepatite A (87\%), febre amarela (46\%), tétano e difteria (dT) (36\%), sarampo, caxumba e rubéola (28\%), poliomielite (19\%), vacina antimeningocócica C (6\%), influenza (2\%) e a vacina da raiva foi recomendada para $3 \%$ destes viajantes. A profilaxia da malária foi recomendada para $86(87,8 \%)$ indivíduos atendidos, sendo 55 com cloroquina e $31 \mathrm{com}$ doxiciclina. $\mathrm{O}$ tratamento autoadministrado para diarréia do viajante foi indicado para 97 (99\%) dos viajantes, sendo a medicação de escolha o ciprofloxacino. Dos 98 voluntários atendidos, 14 viajaram $(14,2 \%)$ e $8(8,2 \%)$ não foi obtida informação sobre a viagem. A média de dias de permanência foi de 19,7 $($ mínimo=6; máximo=43) dias. A profilaxia da malária foi indicada para dez destes viajantes, dois viajantes referiram adesão à indicação da quimioprofilaxia da malária de forma correta, seis pararam a medicação assim que retornaram ao Brasil e dois não tomaram a medicação indicada. Nenhum paciente teve malária durante ou após a viagem. Treze (93\%) dos pacientes utilizaram o repelente contra mosquitos. Três viajantes referiram diarreia, um apresentou infecção de vias aéreas superiores e um referiu dor abdominal com resolução espontânea.

\section{DISCUSSÃO}

O evento ocorrido no Haiti não foi um acontecimento isolado, sendo frequentes outras catástrofes naturais como as chuvas e deslizamentos ocorridos em janeiro de 2011 no Rio de Janeiro, as enchentes no nordeste do Brasil no mesmo período e o terremoto seguido de tsunami no Japão em março de 2011, e que mobilizaram manifestações de ajuda humanitária. A prevenção dos riscos de adoecimento durante as viagens é um dos objetivos da MV, independente dos objetivos da viagem, como no nosso caso o relato das medidas recomendadas aos viajantes envolvidos em ajuda humanitária no Haiti.

Em situações de desastres naturais existe o risco de emergências em saúde pública, não somente às vítimas, mas também aos indivíduos que se dedicam a ajudar voluntariamente. Outro aspecto a ser considerado é que nem sempre estes indivíduos recebem orientação antes de se deslocar para estas áreas, ou quando o fazem não há intervalo de tempo o suficiente para implantação das medidas necessárias, como por exemplo, o cumprimento adequado de esquemas vacinais ${ }^{11,12}$.

Nosso relato é diferente do observado por Küpper et al. ${ }^{11}$ em que a média de idade foi de 19 anos, portanto indivíduos jovens, pertencentes a grupo etário que parece menos aderente às medidas de prevenção recomendadas. O reforço de tétano e difteria (dT) foi indicado a todos os que apresentavam a última dose há mais de 5 anos (36\%), pelo risco de ferimentos devido às precárias condições locais. A vacina de poliomielite foi indicada para proteção individual e coletiva, uma vez que havia possibilidade de reintrodução da doença no Haiti pelos voluntários provenientes de regiões onde a doença é endêmica. $\mathrm{O}$ reforço de vacina contra sarampo, caxumba e rubéola foi indicado para aqueles que não comprovaram duas doses. Já as vacinas contra febre tifóide e hepatite A foram indicadas devido às precárias condições de saneamento e higiene. A vacina contra hepatite $B$ foi indicada a todos que não haviam recebido previamente devido ao risco de contato com materiais biológicos na ajuda aos feridos, mesmo por aqueles que não eram profissionais de saúde. A vacina contra febre amarela foi indicada devido ao fato de o Haiti exigir a vacinação de pessoas provenientes de países endêmicos para a doença. Vacina contra Meningite $\mathrm{C}$ foi indicada pelo risco ocupacional (6\%), assim como contra Influenza (2\%), e para que os viajantes não fossem fonte de infecção no retorno. A vacina anti-pneumocócica 23 valente foi indicada para o viajante com mais de 60 anos de 
idade, como é rotina no Programa Nacional de Imunizações (PNI). Vacina contra raiva foi pouco indicada devido ao período curto entre a consulta e a viagem, impossibilitando a administração do esquema pré-exposição completo que necessita de um intervalo de 21 dias.

O Haiti é um país endêmico para malária, predominantemente por Plasmodium falciparum sensível a cloroquina ${ }^{2}$. A adesão à quimioprofilaxia da malária pelos viajantes contactados foi baixa, semelhante ao que é observado em outros estudos ${ }^{13,14}$. A baixa adesão à quimioprofilaxia é bem descrita nos estudos, mesmo naqueles em que a orientação pré-viagem é realizada de forma adequada ${ }^{10}$. Devido às precárias condições de alojamento e do sistema de saúde local, a quimioprofilaxia da foi recomendada para $86(87,8 \%)$ indivíduos atendidos, sendo 55 com cloroquina e 31 com doxiciclina. O tratamento autoadministrado para diarreia do viajante foi

\section{REFERÊNCIAS}

1. World Tourism Organization (UNWTO). UNWTO Tourism Highligths, 2013 edition. New York; 2013 [Cited 2014 March 4]. Available from: http://www.unwto.org/facts/eng/ barometer.htm.

2. Zuckerman JN. Travel medicine. BMJ. 2002;325:260-4. doi: 10.1136/bmj.325.7358.260.

3. Igreja RP. Travel medicine: a new field of work for the specialist in infectious and parasitic diseases. Rev Soc Bras Med Trop. 2003;36(4):539-40. http://dx.doi.org/10.1590/ S0037-86822003000400020.

4. Mascheretti M, Pierrotti LC, Chaves TSS. Medicina de viagem. In: Martins MA, Carrilho FJ, Alves VAF, Castilho EA, Cerri GG, Wen CL, editores. Clínica médica. Barueri: Manole; 2009. v.7, p.696-705.

5. Gautret P, Freedman DO. Travel medicine, a speciality on the move. Clin Microbiol Infect. 2010;16(3):201-2. doi: 10.1111/j.1469-0691.2009.03134.x.

6. Schlagenhauf P, Santos-O'Connor F, Parola P. The practice of travel medicine in Europe. Clin Microbiol Infect. 2010;16(3):203-8. doi: 10.1111/j.1469-0691.2009.03133.x.

7. LaRocque RC, Jentes ES. Health recommendations for international travel: a review of the evidence base of travel medicine. Curr Opin Infect Dis. 2011;24(5):403-9. doi: 10.1097/QCO.0b013e32834a1aef.

8. Chinwa LS, Mascheretti M, Chaves T do S, Lopes MH. Travelers' vaccinations: experience from the Travelers' Clinic of Hospital das Clínicas, University of São Paulo School of Medicine. Rev Soc Med Trop. 2008;41(5):474-8. http://dx.doi.org/10.1590/S0037-86822008000500008. indicado para 97 (99\%) dos viajantes, sendo a medicação de escolha o ciprofloxacino.

Um dos papéis da medicina de viagem (MV) nos desastres naturais é o de colaborar com as ações de vigilância em saúde em fornecer resposta rápida e efetiva na orientação pré-viagem de viajantes que prestarão ajuda humanitária em áreas críticas e acometidas pelos desastres naturais. Esta ação visa reduzir os riscos de adoecimento no local de destino e que essas pessoas sejam fonte de doenças ao retornarem aos seus locais de origem.

A medicina de viagem se faz presente a cada dia entre nós e ultrapassa barreiras em situações inesperadas como de emergências e respostas rápidas em saúde pública. É importante ressaltar que hoje o viajante vai além das fronteiras de seus objetivos pessoais, e participa de forma voluntária de ações desafiantes como ajudas humanitárias em situações de emergência e em condições desconhecidas.

9. Chaves, TSS, Mascheretti, M, Alves, JR, Boulos, M, Lopes, MH. Travel medicine in the state of São Paulo, Brazil. Travel Med Infect Dis. 2012;10(5-6):283-4. doi: http:// dx.doi.org/10.1016/j.tmaid.2012.09.005.

10. Carlson LC, Hirshon JM, Calvello EJB, Pollak AN. Operative care after the Haiti 2010 earthquake: Implications for post-disaster definitive care. Am J Emergency Med. 2013;31(2):429-31. 2013. http://dx.doi.org/10.1016/j. ajem.2012.10.020.

11. Küpper T, Rieke B, Neppach K, Morrison A, Martin J. Health hazards and medical treatment of volunteers aged 18 and 30 years working in international social projects of non-governmental organizations (NGO). Travel Med Infect Dis. 2014;12:385-95. doi: 10.1016/j.tmaid.2013.11.004.

12. Costa M. Pre-deployment, travel health advice for humanitarian health workers: helping the helpers. Travel Med Infect Dis. 2014;12:543-4. doi: 10.1016/j. tmaid.2014.08.001.

13. Dahlgren AL, Deroo L, Avril J, Bise G, Loutan L. Health risks and risk-taking behaviors among International Committee of the Red Cross (ICRC) expatriates returning from humanitarian missions. J Travel Med. 2009;16:382-90. doi: 10.1111/j.1708-8305.2009.00350.x.

14. Laver SM, Wetzels J, Behrens RH. Knowledge of malaria, risk perception, and compliance with prophylaxis and personal and environmental preventive measures in travelers exiting Zimbabwe from Harare and Victoria Falls International Airport. J Travel Med. 2001;8:298-303. doi: 10.2310/7060.2001.23975. 